\title{
Automated Classification of Quilt Photographs Into Crazy and Non- crazy
}

\author{
Alhaad Gokhale and Peter Bajcsy ${ }^{\mathrm{a}}$ \\ ${ }^{a}$ Dept. of Computer Science and Engineering, Indian institute of Technology, Kharagpur, India; \\ bational Center for Supercomputing Applications, Univ. of Illinois at Urbana-Champaign
}

\begin{abstract}
This work addresses the problem of automatic classification and labeling of 19th- and 20th-century quilts from photographs. The photographs are classified according to the quilt patterns into crazy and non - crazy categories. Based on the classification labels, humanists try to understand the distinct characteristics of an individual quilt-maker or relevant quilt-making groups in terms of their choices of pattern selection, color choices, layout, and original deviations from traditional patterns. While manual assignment of crazy and non-crazy labels can be achieved by visual inspection, there does not currently exist a clear definition of the level of crazy-ness, nor an automated method for classifying patterns as crazy and non-crazy.

We approach the problem by modeling the level of crazy-ness by the distribution of clusters of color-homogeneous connected image segments of similar shapes. First, we extract signatures (a set of features) of quilt images that represent our model of crazy-ness. Next, we use a supervised classification method, such as the Support Vector Machine (SVM) with the radial basis function, to train and test the SVM model. Finally, the SVM model is optimized using N-fold cross validation and the classification accuracy is reported over a set of 39 quilt images.
\end{abstract}

Keywords: Quilt Classification, crazy, non-crazy, texture discrimination, texture symmetry

\section{INTRODUCTION}

With the advancements in camera-based imaging, humanities researchers have been imaging very large collections of historical artifacts that generate unprecedented amounts of digital data. These image collections have to be stored, organized, annotated, classified, and searched in order to serve humanities researchers. One example of such an image collection is the repository of the photographs of the 19th- and 20th-century quilts. This work tackles the problem of automatic classification and labeling of the photographs of the 19th- and 20th-century quilts with a specific focus on classification of quilts into those with crazy and non - crazy patterns.

In general, automatic classification over a large collection of quilt photographs (150,000 in the Matrix database) allows humanities researchers to search and interpret distinct characteristics of an individual quilt-maker or a group of quiltmakers. Understanding of these characteristics can reveal the mappings between the choices of layout, pattern, color, or shapes, and the affiliations of quilters in terms of their religious and political beliefs or social standing. The challenge of automatic classification lies in the fact that the assignment of classification labels has been done so far manually based on visual inspection without a mathematical model. The motivation of our work is to define a non-existent mathematical model for the assignment of the level of crazy-ness, and develop a classification algorithm to remove the manual assignment process.

Previous work has focused to document, preserve and share this cultural heritage. The previous work available can be viewed as two extremes: one by humanist focusing on interpretation of characteristics of quilts; the other in the field of texture analysis and synthesis in statistics and computer vision. Julesz suggested that two texture images will be perceived by human observers to be the same if some appropriate statistics of these images match. This suggests that the two main tasks in statistical texture discrimination are (1) picking the right set of statistics to discriminate, (2) finding an algorithm that discriminates them. In comparison to the previous work, this work suggests an approach to extract statics that mathematically model the presence of global symmetry for quilt patches.

Computer Vision and Image Analysis of Art II, edited by David G. Stork, Jim Coddington, Anna Bentkowska-Kafel, Proc. of SPIE Vol. 7869, 78690M - (c) 2011 SPIE · CCC code: 0277-786X/11/\$18 - doi: 10.1117/12.876370 
According to our knowledge, there does not exist currently a clear definition of the level of crazy-ness, nor an automated method for classifying patterns as crazy and non-crazy. The current annotation method is manual and the assignment of crazy and non-crazy labels is achieved by visual inspection.

Our approach is to model the level of crazy-ness based on the distribution of clusters of color-homogeneous connected image segments of similar shapes. The model is obtained by performing segmentation into color-homogeneous regions, describing each segment with its color statistics and shape statistics, and clustering regions based on these statistics. Each quilt image is then represented by the parameters of all existing clusters in the image (the signature). Finally, quilt images are classified into crazy and non-crazy by building a supervised support vector machine (SVM) classifier. The SVM classifier is optimized using 10 -fold cross validation and the classification accuracy is reported over a set of 40 quilt images.

The image classification into crazy and non-crazy pattern is described by introducing quilt image signature that captures hierarchical structure of quilt patterns in Section 2. Section 3 presents the design of the classification algorithm followed Section 4 with experimental classification results. We summarize our work in Section 5.

\section{CHARACTERIZATION OF QUILT PATTERNS}

\subsection{Introduction}

In order to classify quilt patterns, one has to characterize the hierarchical structure of content in quilt photographs. The characterization is based on our observations of human visual inspection where parts of the quilt pattern are grouped in a hierarchical order. These hierarchical groupings can be characterized with quilt image signature. The quilt signatures reflect the level of symmetry and repetitiveness in patterns obtained by hierarchical groupings. Quilt signatures (or quilt image features) are extracted by detecting (1) color homogeneous texels (low level image features), and (2) shape homogeneous classes of texels (medium level image features). The assignment of crazy and non-crazy labels based on quilt signatures would be viewed as the high level image feature.

\subsection{Color Homogeneous Texels}

A texel is a collection of color homogeneous connected pixels that define the smallest unit of interest. In order to detect texels and capture the low level spatial arrangement of color homogeneous regions, we identify connected pixels having same/similar color as illustrated in Figure 1. Texel detection is achieved by first identifying same / similar color pixels and then by color discretization using k-means clustering algorithm over the red-green-blue (RGB) color space. Cluster centers are hence identified from the algorithm and each pixel is associated with its corresponding cluster center based on its Euclidian distance in the RGB color space. Thus, first a mapping is produced from the quilt image to an image map where each pixel corresponds to the cluster center it is associated with. Then connected components are identified in the image map using 4-nearest neighbor blob coloring algorithm. Hence, a set of texels is obtained where each texel is color homogeneous connected region. Each texel represents patch of quilt.

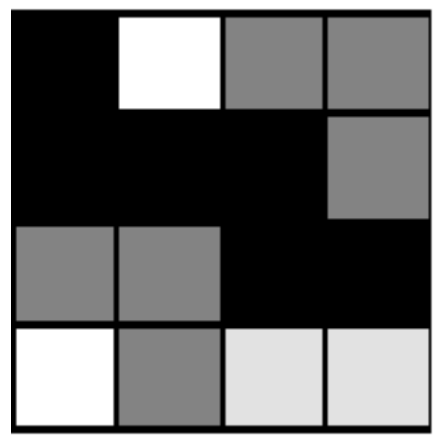

(a)

\begin{tabular}{|c|c|c|c|}
\hline 1 & 3 & 2 & 2 \\
\hline 1 & 1 & 1 & 2 \\
\hline 2 & 2 & 1 & 1 \\
\hline 3 & 2 & 3 & 3 \\
\hline
\end{tabular}

(b)

\begin{tabular}{|c|c|c|c|}
\hline 1 & 2 & 3 & 3 \\
\hline 1 & 1 & 1 & 3 \\
\hline 4 & 4 & 1 & 1 \\
\hline 5 & 4 & 6 & 6 \\
\hline
\end{tabular}

(c)

Figure 1. Figure illustrating detection of color homogeneous texels. (a) The input is a $4 \times 4$ grid of pixels with each pixel having an intensity(color) value (b) The four unique intensity values are mapped to 3 clusters obtained using k-means with $\mathrm{k}=3$ (c) Six texels are obtained by identifying cluster blobs corresponding to same cluster. 


\subsection{Shape Homogeneous Classes of Texels}

After detecting texels in quilt images, we have to characterize spatial distribution and symmetry of texels of similar shape and size. The descriptors of spatial arrangement and distribution of texels correspond to the next higher level of hierarchical characterization of quilt patterns. Thus, a quilt pattern model includes statistical parameters of the spatial distribution of color-similar texels. The detection of shape homogeneous classes of texesl is executed in a bottom up manner. Each texel is put into a class of its own and the classes are iteratively merged based on similarity in shape of containing texels. Shapes are determined to be similar based on similar measures of perimeter and area contained by the texel.

\subsection{Quilt Signature}

A quilt signature is a set of quantifiable features derived from the presence and spatial distribution of a class of texels. The signature becomes the input to a supervised classification method in order to determine the level of crazy-ness.

The features forming a quilt signature are: (1) Number of Pixels, (2) Number of Classes, (3) Number of Classes identified as Noise, (4) Number of Classes containing single texel, (5) Number of Classes containing two texels, (6) Number of Classes containing multiple texels, (7) Maximum number of texels for any class, (8) Minimum variance in texel location for any class, (9) Minimum value of deviation from symmetry $(\mu-\mathrm{M}) / \sigma$ where $\mu, \mathrm{M}$ and $\sigma$ are expected value, most frequent value and standard deviation of the intra-class inter-texel distance respectively.

\section{ALGORITHMIC DESIGN}

\subsection{Introduction}

The classification methodology consists of four steps. In the first step, a color-homogeneous Region or Texel is detected by color based K-means clustering followed by connectivity analysis. This step leads to a cluster of color homogeneous regions that represent quilt patches with similar colors. The second step uses a divide-and-conquer approach to identify sub-clusters that share similar shape properties such as area and perimeter. For each sub-cluster, statistics of nearest neighbor distances are computed for example, the mean and variance of distances. In the third step, a quilt signature per image is formed from parameters including the number of clusters containing a single colorhomogeneous region/texel, the maximum and average number of color-homogeneous regions per cluster of regions, the minimum variance present in any class, etc. Our selection of these parameters is based on the observation that crazy patterns have a small number of color-homogeneous and shape-similar regions in a cluster and a large number of clusters containing only a single region. They also have no symmetry and hence large variance in inter - Region nearest neighbor distance. In contrary, non-crazy patterns will have a small number of clusters and a large number of color-homogeneous and shape-similar regions in a cluster. In the last step, a Support Vector Machine (SVM) model is trained using labeled quilt images and cross validated by 50:50 train: test split.

In a nutshell, the algorithm designed consists of quilt signature extraction and classification steps. The input is an image and the output is a label (crazy or non-crazy). The processing sequence can be described by the following workflow:

color based image segmentation $\rightarrow$ connectivity analysis $\rightarrow$ shape similarity analysis $\rightarrow$ signature extraction $\rightarrow$ statistical learner

These processing steps are described next.

\subsection{Color Based Image Segmentation}

The goal of image segmentation is to identify pixels having similar color by clustering pixel intensities in a color space and assigning each pixel a cluster. Segmentation is accomplished by using the k-means clustering algorithm to identify color clusters mapped into the RGB space. The control parameter ' $\mathrm{k}$ ' which is the number of clusters required is 
determined heuristically to achieve optimal performance. The rule for choosing " $\mathrm{k}$ " follows an observations that a very large value of ' $k$ ' may lead to over-fitting noise while a very small value may lead to ineffective image model.

\subsection{Connectivity Analysis}

The goal of connectivity analysis within clusters is to obtain image segments corresponding to quilt patches. These color homogeneous connected regions are the smallest units of statistical significance in our algorithm. The connectivity analysis is done using 4-nearest neighbor blob coloring method and leads to color homogeneous texels.

\subsection{Shape Similarity}

The goal of shape similarity analysis is to identify a quilt pattern as a layer of texture with similar color and shape properties of texels. This class of shape homogeneous texels is obtained by comparing perimeter and areas of texels and consequently merging them hierarchically.

A class is defined as a set of texels which are similar in shape and size. First, each texel is put in a class of its own. Then classes are merged hierarchically in an iterative fashion according to the average area and perimeter of the texels it contains i.e. two classes are merged if they lie with the threshold of similarity. The parameters are updated at each iteration. Finally, the statistical features of the class are evaluated. These statistical features are those required for computing the signature.

\subsection{Signature Extraction}

The quilt features which have been described in section 2.4 are extracted by evaluating the parameters globally from each of the classes iteratively.

\subsection{Statistical Learner}

The goal of statistical learner, such as Support Vector Machine (SVM) method with the radial basis function kernel, is to model the mapping between the signature (input) and the level of crazy-ness (output). The training is optimized using 10 -fold cross validation. The SVM model can be used to classify unlabeled images.

\section{EXPERIMENTAL RESULTS}

\subsection{Test Data and Experimental Setup}

We evaluated the capability of our algorithm on quilt photographs available at the MATRIX quilt database. Forty test images were selected manually out of 150,000 available quilt images. The manual process allowed elimination of low quality quilt photographs due bad lighting conditions, folded quilts, or presence of foreign objects in the photograph.

The processing sequence started with extracting quilt signatures that were used to train a support vector machine (SVM) classifier/learner using a radial basis function kernel. The SVM classifier was optimized using 10-fold cross validation and the classification accuracy was reported over a set of 40 images.

\subsection{Algorithmic Control Parameters}

Figure 2 and Figure 3 show the effects of the clustering parameter (number of cluster) and texel detection parameter (color thresholding) on the features extracted over the hierarchical structure of the image. 


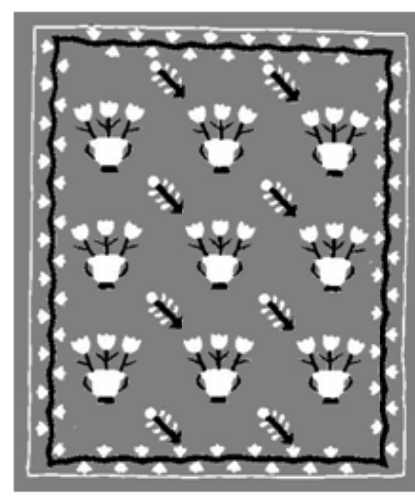

$\mathrm{K}=3$

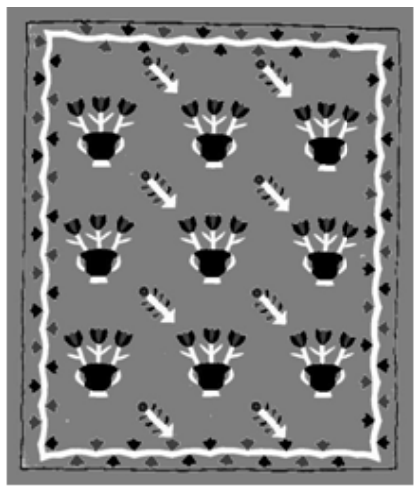

$K=5$

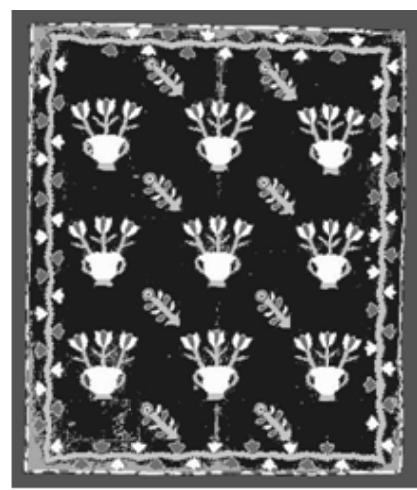

$K=10$

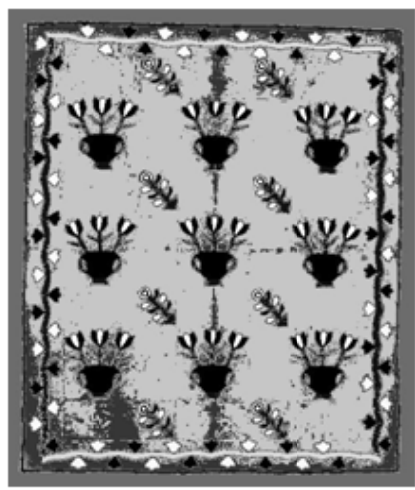

$K=15$

Figure 2. The effect of the clustering parameter " $\mathrm{k}$ " on the clusters formed. For low-moderate values of $\mathrm{k}=3$ and $\mathrm{k}=5 \mathrm{clusters}$ are quickly computed whereas large $\mathrm{k}$ values like $\mathrm{k}=10$ and $\mathrm{k}=15$ take time to compute and also lead to over-fitting.

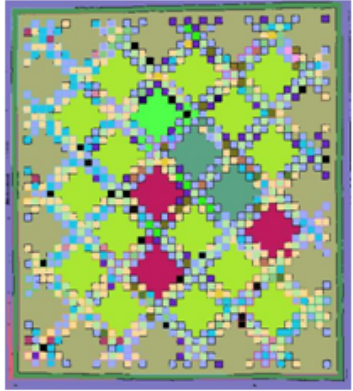

Low Threshold 5\%

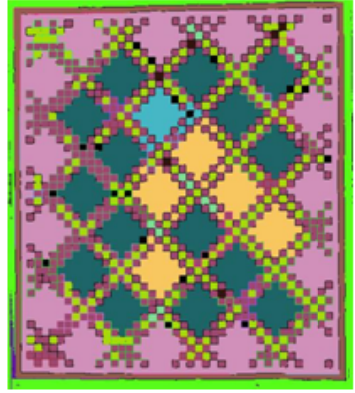

Moderate Threshold $10 \%$

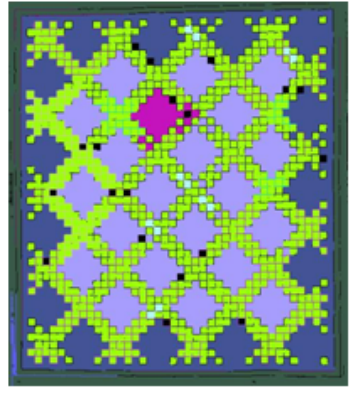

Moderately High $15 \%$

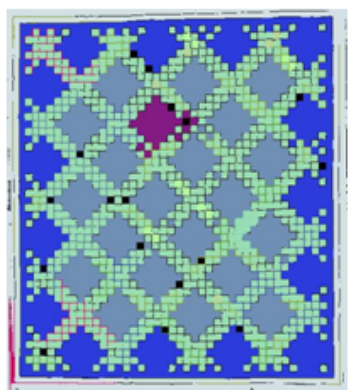

High Threshold $20 \%$

Figure 3. Similarity threshold is that tolerance value over which two shapes can be determined to be similar. Figure clearly shows that a small threshold leads to error improving when the threshold is increased.

\subsection{Comparison of Crazy and Non-Crazy Quilt Signature Parameters}

Figure 4 and Figure 5 show non-crazy and crazy quilts and the results of the quilt signature extraction. The quilt signatures for these two images indicate the following principle differences (1) the non-crazy quilt has a small number of classes with a large number of texels whereas crazy quilts do not posses such a distribution, (2) the crazy quilt contains a large number of classes containing a single texel and (3) there is high inter-texel deviation from symmetry within classes of crazy quilts.

Table 1. The normalized signature of quilts shown in Figure 4 and Figure 5.

\begin{tabular}{llllllllllll}
\hline Figure & $\begin{array}{l}\text { No. of } \\
\text { pixels }\end{array}$ & $\begin{array}{l}\text { No. of } \\
\text { Classes }\end{array}$ & $\begin{array}{l}\text { No. of } \\
\text { Classes } \\
\text { (Noise) }\end{array}$ & $\begin{array}{l}\text { No. of } \\
\text { classes } \\
\text { (One } \\
\text { texel) }\end{array}$ & $\begin{array}{l}\text { No. of } \\
\text { classes } \\
\text { (Two } \\
\text { texels) }\end{array}$ & $\begin{array}{l}\text { No. of } \\
\text { classes } \\
\text { (Multiple } \\
\text { texels) }\end{array}$ & $\begin{array}{l}\text { Maximum } \\
\text { class size }\end{array}$ & $\begin{array}{l}\text { Minimum } \\
\text { Variance }\end{array}$ & $\begin{array}{l}\text { Minimum } \\
\text { value of } \\
(\boldsymbol{\mu}-\mathbf{M}) / \boldsymbol{\sigma}\end{array}$ \\
\hline $\begin{array}{l}\text { Figure } \\
\mathbf{4}\end{array}$ & 260700 & 56 & 52 & 2 & 0 & 2 & 24 & 1 & 1 & $\begin{array}{l}\text { Typen- } \\
\text { crazy }\end{array}$ \\
$\begin{array}{l}\text { Figure } \\
\mathbf{5}\end{array}$ & 329600 & 301 & 118 & 98 & 49 & 36 & 2 & 100 & 100 & Crazy \\
\hline
\end{tabular}




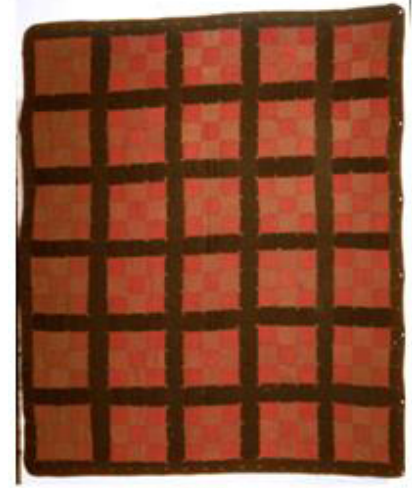

(a)

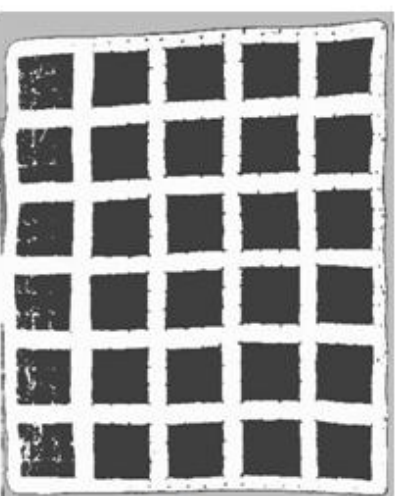

(b)

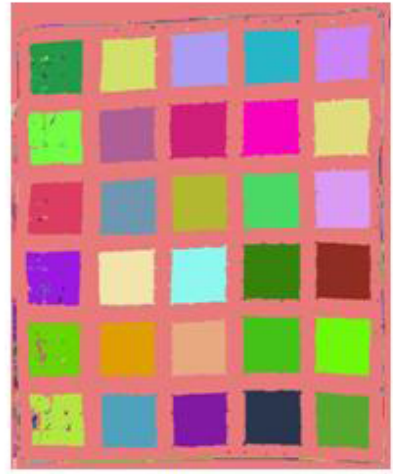

(c)

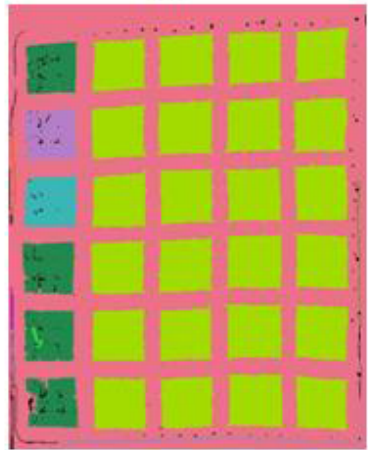

(d)

Figure 4. A visual form of the physical stages of the algorithm. (a)Shown is a non-crazy quilt photograph input to the algorithm. (b)The result after k-means clustering, where each cluster center is represented by a shade of grey. (c)The result after blob coloring implementation where each shade corresponds to a texel or quilt patch. (d)A visual form in which each patch of similarly shaped quit is given the same shade of grey.
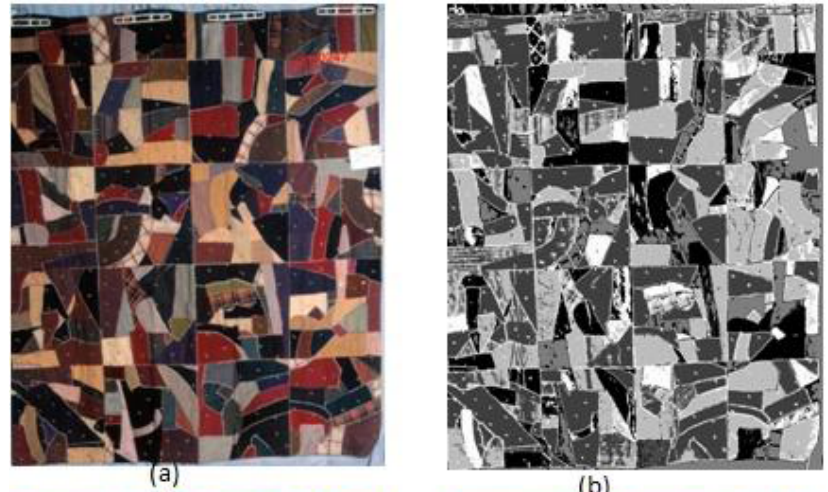

(b)

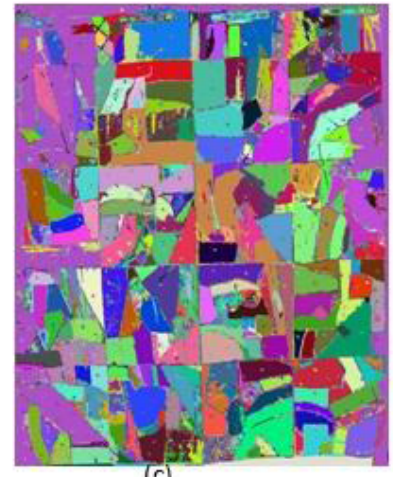

(c)

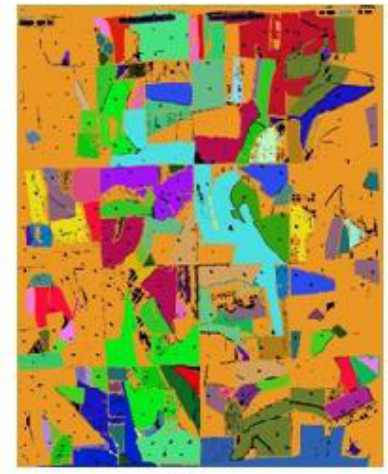

(d)

Figure 5. A visual form of the physical stages of the algorithm. (a)Shown is a crazy quilt photograph input to the algorithm. (b)The result after k-means clustering, where each cluster center is represented by a shade of grey. (c)The result after blob coloring implementation where each shade corresponds to a texel or quilt patch. (d)A visual form in which each patch of similarly shaped quit is given the same shade of grey.

\subsection{Observed Accuracy}

The SVM classifier led to a model with an accuracy of about $90 \%$ over a set of 40 quilt images.

\subsection{Time Complexity}

The algorithm has a linear time complexity $\mathrm{O}(\mathrm{n})$ with the number of pixels (assuming algorithm control parameters are fixed). 


\subsection{Data Visualization}

The high dimensional data was mapped to 2 dimensions using the principle component analysis algorithm for visualization of data distribution. It clearly shows a separation in trend for the parameters extracted by the algorithm along the direction of maximum co-variance.

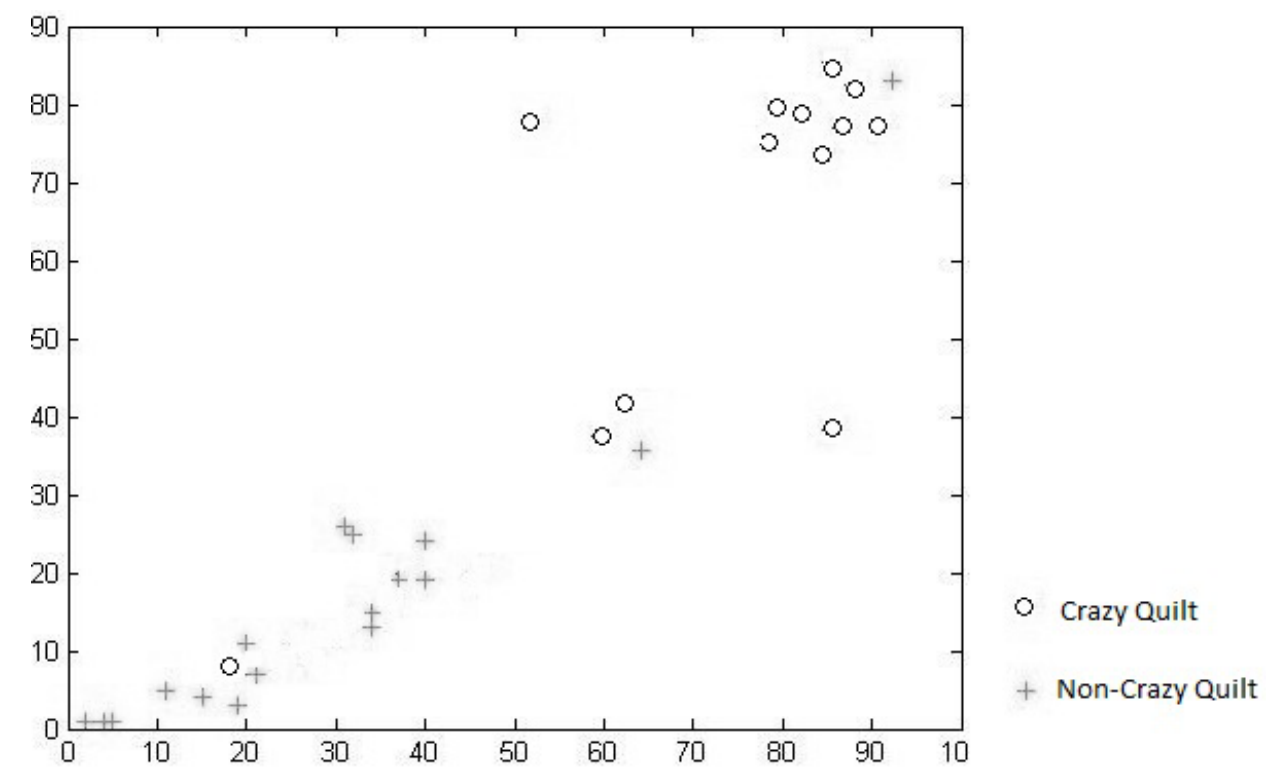

Figure 6. The high dimensional data was mapped to 2 dimensions using the principle component analysis algorithm for visualization of data distribution. The horizontal axis is an axis in space corresponding to the direction of maximum co-variance. The vertical axis corresponds to the direction of next largest value.

\section{SUMMARY}

We implemented the classification methodology using a combination of Java and Matlab code. The algorithm was applied to 39 quilt images from the MATRIX database at the Michigan State University. We report almost 90 percent classification accuracy over 39 images using SVM and its radial basis function. In the future, we plan on extending the categorical model of crazy-ness to a continuous function reflecting the level of craziness.

\section{REFERENCES}

[1] K. Popat and R. W. Picard, "Novel cluster probability models for texture synthesis, classification, and compression," in Proc. SPIE Visual Communication and Image Proc., vol. 2094, (Boston), pp. 756-768, Nov. 1993.

[2] Bela Julesz, "Visual pattern discrimination," IRE Transactions on Information Theory, 8(2):84-92, 1962

[3] R. O. Duda and P. E. Hart, "Pattern classification and scene analysis," Wiley, 1973.

[4] Ballard, D. and Brown, C. (1982), "Computer vision," New Jersey: Prentice Hall.

[5] B. Manjunath, P. Wu, S. Newsam, and II. Shin," A texture descriptor for browsing and similarity retrieval," Signal Processing Image Communication, 2001.

[6] P. Bajcsy and N. Ahuja, "Location- and density-based hierarchical clustering using similarity analysis," IEEE Trans. Pattern Analysis and Machine Intelligence, vol. 20, no. 9, pp. 1011-1015, 1998. 
[7] J. H. Hays, M. Leordeanu, A. A. Efros, and Y. Liu. ,'Discovering texture regularity as a higher-order correspondence problem. In ECCV, volume 2, pages 522-535, 2006.

[8] R. Haralick, "Statistical and structural approaches to texture," Proc. IEEE, vol. 67, pp. 786-804, May 1979.

[9] Quilt Culture: Tracing the Pattern. Cheryl B. Torsney and Judy Elsley, eds. Columbia/London: University of Missouri Press. 69-84.

[10] Hartigan, J. A.; Wong, M. A. (1979). "Algorithm AS 136: A K-Means Clustering Algorithm". Journal of the Royal Statistical Society, Series C (Applied Statistics) 28 (1): 100-108.

[11] M. Petrou and P. Bosdogianni, Image Processing the Fundamentals, Wiley, UK, 2004.

[12] A. Gorban, B. Kegl, D. Wunsch, A. Zinovyev (Eds.), Principal Manifolds for Data Visualisation and Dimension Reduction, LNCSE 58, Springer, Berlin - Heidelberg - New York, 2007. 